\title{
Sunspot group tilt angle measurements from historical observations
}

\author{
V. Senthamizh Pavai, R. Arlt* \\ Leibniz Institute for Astrophysics Potsdam (AIP), An der Sternwarte 16, 14482 Potsdam, Germany
}

A. Diercke

AIP; Institut für Physik und Astronomie, Universität Potsdam, Karl-Liebknecht-Str. 24/25, 14476 Potsdam, Germany

C. Denker

Leibniz Institute for Astrophysics Potsdam (AIP), An der Sternwarte 16, 14482 Potsdam, Germany

J. M. Vaquero

Dept. de Física, Centro Universitario de Mérida, Universidad de Extremadura, Avda Santa Teresa de Jornet 38, 06800 Mérida, Spain

\section{Abstract}

Sunspot positions from various historical sets of solar drawings are analysed with respect to the tilt angles of bipolar sunspot groups. Data by Scheiner, Hevelius, Staudacher, Zucconi, Schwabe, and Spörer deliver a series of average tilt angles spanning a period of 270 years, additional to previously found values for $20^{\text {th }}$-century data obtained by other authors. We find that the average tilt angles before the Maunder minimum were not significantly different from the modern values. However, the average tilt angles of a period 50 years after the Maunder minimum, namely for cycles 0 and 1, were much lower and near zero. The normal tilt angles before the Maunder minimum suggest that it was not abnormally low tilt angles which drove the solar cycle into a grand minimum.

Keywords: Sun: sunspots; tilt angles; cycle-averaged tilt angle

\section{Introduction}

The long-term study of solar cycle properties using historical observations has provided mainly the sunspot number (Clette et al., 2014). However, we can also ac'cess other properties more directly related to the solar dynamo through historical sunspot drawings. The tilt angle of sunspot groups is among those parameters and can be included in certain types of dynamo models. Bipolar sunspot groups exhibit an axis through the two main magnetic polarities. The tilt angle is the angle at which this axis is orientated with respect to the solar equator. It is an important property in flux-transport dynamos (Babcock-Leighton dynamos, see e.g. Charbonneau, 2010, Sect. 4.8) in which it provides the source term for the poloidal magnetic field which in turn correlates with the strength of the next cycle. The tilt angles are widely believed to be the result of buoyantly unstable magnetic flux tubes at the bottom of the convection zone, rising under the influence of rotation, internal twist, and magnetic tension.

According to results of the thin flux tube approximation, tilt angles are either due to writhing of rising flux

\footnotetext{
* Corresponding author

Email address: svalliappan@aip.de (V. Senthamizh Pavai, R. Arlt)
}

loops by the Coriolis force or the pitch angle of the subsurface field wound up by the differential rotation (D'Silva \& Choudhuri, 1993). The combination of magnetic buoyancy and the Coriolis force generates the correct latitudinal distribution of tilt angles, according to numerical simulations in the thin flux tube framework (e.g. D'Silva \& Choudhuri, 1993; Caligari et al., 1995; Fan \& Fisher, 1996; Weber et al., 2013). These computations can also reproduce the correlation between the magnetic field strength and the tilt angle which is seen in some observational studies (Tian et al., 2003; Dasi-Espuig et al., 2010). The average tilt angle and the amplitude of the corresponding cycle appears to be anti-correlated, while the product of the average tilt angle with the cycle amplitude is well correlated with the strength of the following cycle (Dasi-Espuig et al., 2010, 2013).

In thin flux tube models, the tilt angles are even useful in constraining the strength of initial magnetic flux. The strength of the toroidal magnetic field at the bottom of the convection zone has to be in the range of $40-50 \mathrm{kG}$ in order to obey the observed Joy's law (Weber et al., 2011).

Observational studies by Kosovichev \& Stenflo (2008) show that the tilt angles of sunspot groups change gradually over their lifetime except in the beginning of emergence. While the tilt angles are random in the earliest 
Table 1: Mean and median tilt angles for various data sets. Full widths at half-maximum (FWHM) were derived from Gaussian fits to the tilt angle distributions.

\begin{tabular}{lccrrr}
\hline Data source & Years & Mean tilt & Median tilt & FWHM & Number of groups \\
\hline Scheiner & $1618-1627$ & $3.92^{\circ} \pm 0.91^{\circ}$ & $3.63^{\circ} \pm 0.91^{\circ}$ & $33.4^{\circ}$ & 537 \\
Hevelius & $1642-1644$ & $4.79^{\circ} \pm 1.43^{\circ}$ & $5.35^{\circ} \pm 1.43^{\circ}$ & $27.1^{\circ}$ & 130 \\
Staudacher & $1749-1796$ & $2.03^{\circ} \pm 0.98^{\circ}$ & $1.79^{\circ} \pm 0.98^{\circ}$ & $50.3^{\circ}$ & 828 \\
Zucconi & $1754-1760$ & $0.62^{\circ} \pm 2.28^{\circ}$ & $-1.48^{\circ} \pm 2.28^{\circ}$ & $35.8^{\circ}$ & 131 \\
Schwabe & $1825-1867$ & $4.45^{\circ} \pm 0.20^{\circ}$ & $4.69^{\circ} \pm 0.20^{\circ}$ & $40.6^{\circ}$ & 15548 \\
Spörer & $1861-1894$ & $4.86^{\circ} \pm 0.45^{\circ}$ & $4.04^{\circ} \pm 0.45^{\circ}$ & $31.3^{\circ}$ & 2834 \\
\hline
\end{tabular}

phase of emergence, they adjust towards Joy's law during the rest of the emergence phase, i.e. as long as the magnetic flux is growing. It is not straight-forward to draw a direct link between the tilt angles and the emergence of flux tubes in simulations. The average tilt angles are also fairly independent of the cycle phase within fixed latitudinal zones (Li \& Ulrich, 2012).

The study of tilt angles derived for several centuries helps us understand their origin and their relation to the solar cycle. The true tilt angles of sunspot groups are available only from magnetic data of the solar surface for the second half of the $20^{\text {th }}$ century, while pseudo-tilt angles are measured without the polarity information and have a $180^{\circ}$ ambiguity. Pseudo-tilt angles can be computed whenever individual spot positions in sunspot groups are available from drawings or images. They have recently been calculated for the period of 1825-1867 using the sunspots observations by Schwabe (Senthamizh Pavai et al., 2015).

In this paper, we present the tilt angle measurements from further historical sunspot observations, namely the observations by Christoph Scheiner (1618, 1621-1622, 1625-1627), Johannes Hevelius (1642-1644), Johann Caspar Staudacher (1749-1796), Ludovico Zucconi (17541760), and Gustav Spörer (1861-1894).

The details of different solar observations and the methods used in data extraction from those sunspot drawings are described in Sect. 2. The comparison of mean tilt angles and cycle-mean tilt angles from various data are discussed in Sect. 3.

\section{Data set}

Christoph Scheiner started his sunspot observations from Ingolstadt, Germany, in the early 17th century. His first known sunspot drawing was made on 21 October 1611. Most of his data, however, were recorded from Rome. He published observations only for a few days during each of the years of 1611, 1612, 1618, 1621, 1622, and 1624 . In the period 1625-1627, the observations are fairly continuous (drawings covering 342 days in 1625, 163 days in 1626, and 55 days in January-June 1627). His drawings show the sunspot groups traversing the solar disk in a single full-disk drawing (Scheiner, 1630). The positions and areas of the sunspots were measured using 13 circular cursor shapes with areas between one and 364 pixels. The data before 1618 were not included in the tilt angle distribution, because they are extremely coarse and show highly exaggerated sizes of sunspot groups.

Johannes Hevelius recorded his observations of the Sun from Gdańsk, Poland, during the period of 1642-1644 (15 days in October-December 1642, 110 days in MayDecember 1643, and 98 days in January-October 1644). These sunspot drawings were published by Hevelius in an appendix of his book Selenographia (Hevelius, 1647). His drawing style is very similar to Scheiner's style, and the positions and area information were obtained in the same way. It is important to note that these sunspot drawings were made just before the Maunder Minimum, a period of reduced solar activity from 1645 to 1715 approximately (Spörer, 1889; Usoskin et al., 2015).

During the second half of the 18th century Johann Caspar Staudacher (or Staudach) recorded his observations of the Sun from Nuremberg, Germany, and made drawings in 1749-1796. Detailed information about the drawings and the data extraction methods can be found in Arlt (2008) and Arlt (2009). Various methods of estimating the orientation of the drawings had to be employed to measure the sunspot positions. From the data base derived, we use only the spots with quality flags 1 and 2. This basically excludes drawings for which the orientation was estimated using a typical tilt angle for bipolar regions. Quality-3 observations use the tilt as an input in many cases and are therefore not used.

For a rather short time from April 1754 to May 1758 and a short spell in June 1760, Ludovico Zucconi observed the Sun from Venice, Italy, contemporaneously with Staudacher. The positions and areas of individual sunspots were extracted by Cristo et al. (2011) using the HSUNSPOTS tool. The orientation of these drawings were clearly marked by the observer, and we consider them fairly precise.

The drawings of sunspot observations made by Samuel Heinrich Schwabe from Dessau, Germany, in the period 1825-1867, and the extraction of data from them were explained in detail by Arlt (2011) and Arlt et al. (2013). A description of the method that was employed to compute 


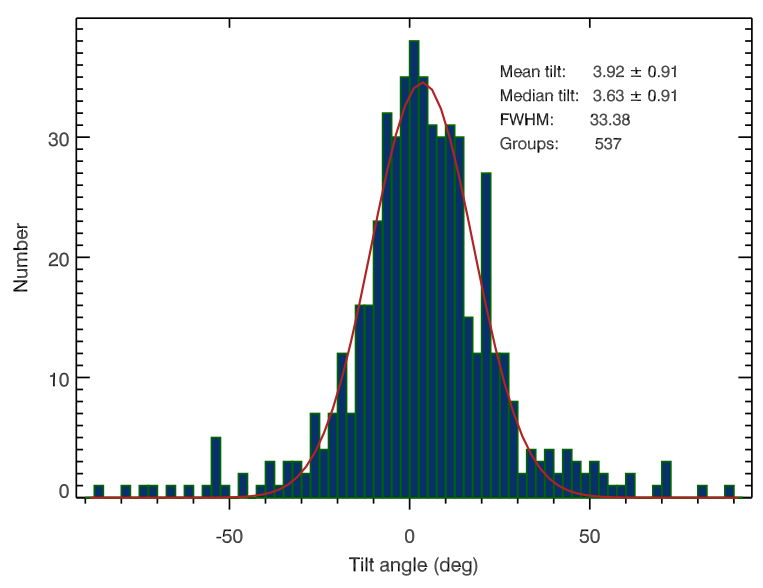

Figure 1: Histogram of the tilt angles of sunspot groups from the drawings by Scheiner. Only groups with area weighted centers within $\pm 60^{\circ} \mathrm{CMD}$ are used. The FWHM was derived from a Gaussian fit (solid line).

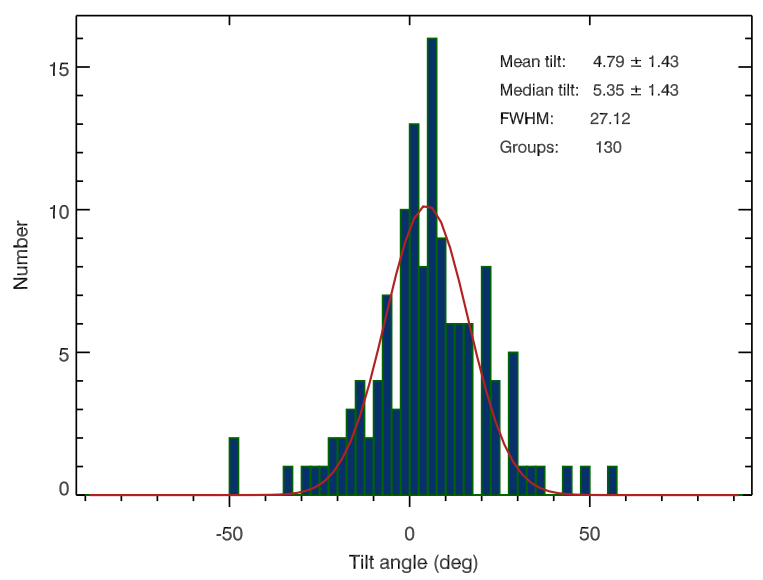

Figure 2: Tilt angle histogram for Hevelius. Only groups with area weighted centers within $\pm 60^{\circ} \mathrm{CMD}$ are used.

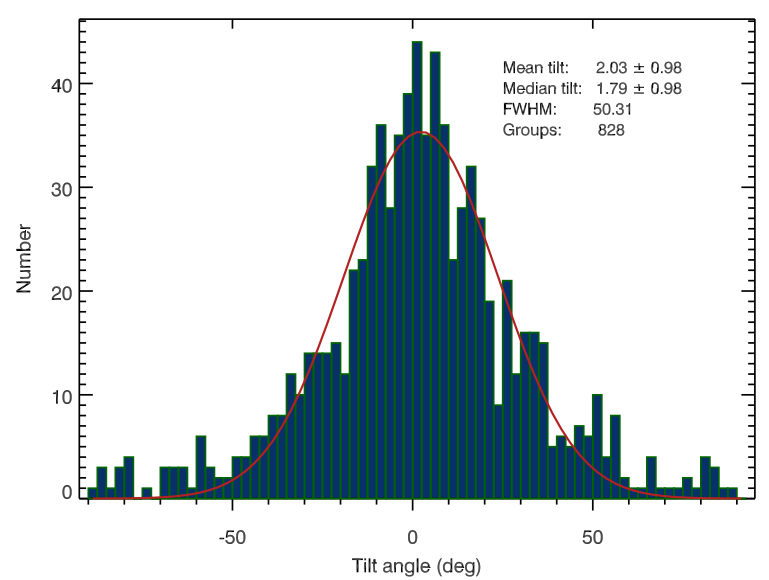

Figure 3: Tilt angle histogram for Staudacher. Only groups with area weighted centers within $\pm 60^{\circ} \mathrm{CMD}$, polarity separations $\Delta \beta>3^{\circ}$, and with quality flags 1 and 2 are used.

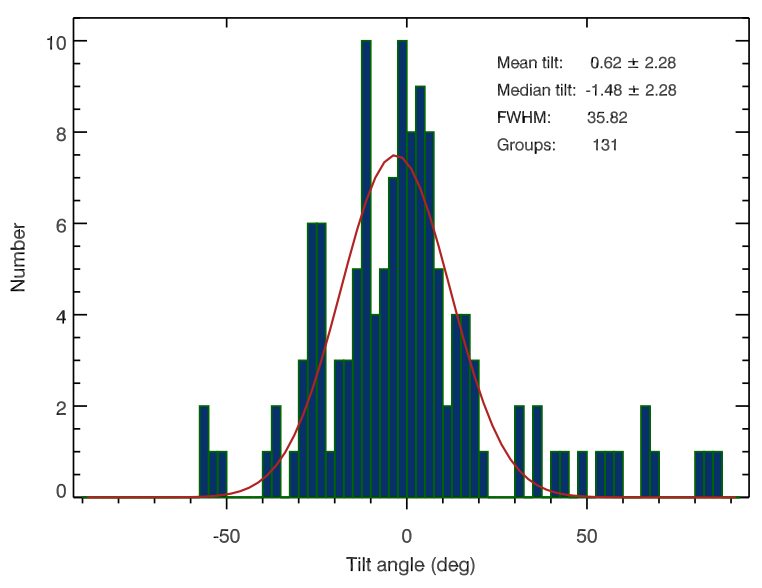

Figure 4: Tilt angle histogram for Zucconi. Only groups with area weighted centers within $\pm 60^{\circ} \mathrm{CMD}$ are used.

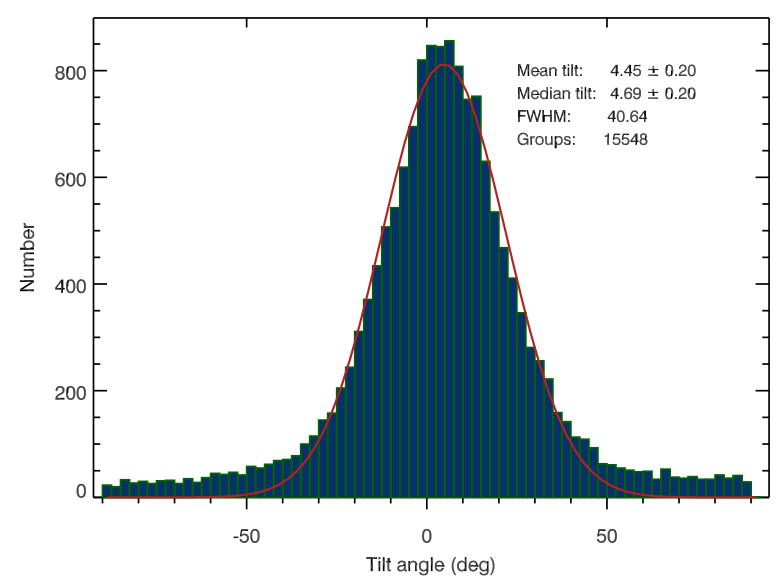

Figure 5: Tilt angle histogram for Schwabe. Only groups with area weighted centers within $\pm 60^{\circ} \mathrm{CMD}$ and polarity separations $\Delta \beta>3^{\circ}$ are used.

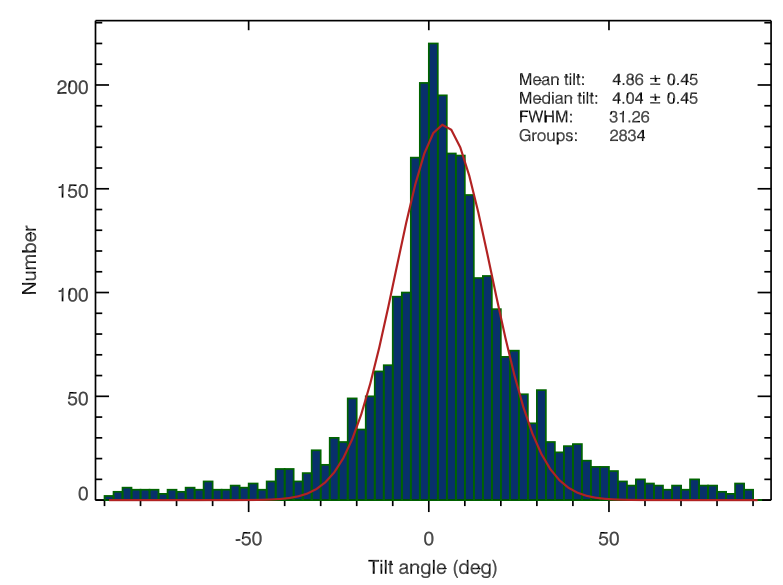

Figure 6: Tilt angle histogram for Spörer and groups with polarity separations $\Delta \beta>3^{\circ}$. Only spots with areas $\geq$ $1 \mathrm{MSH}$ were considered while calculating the tilt angle. 

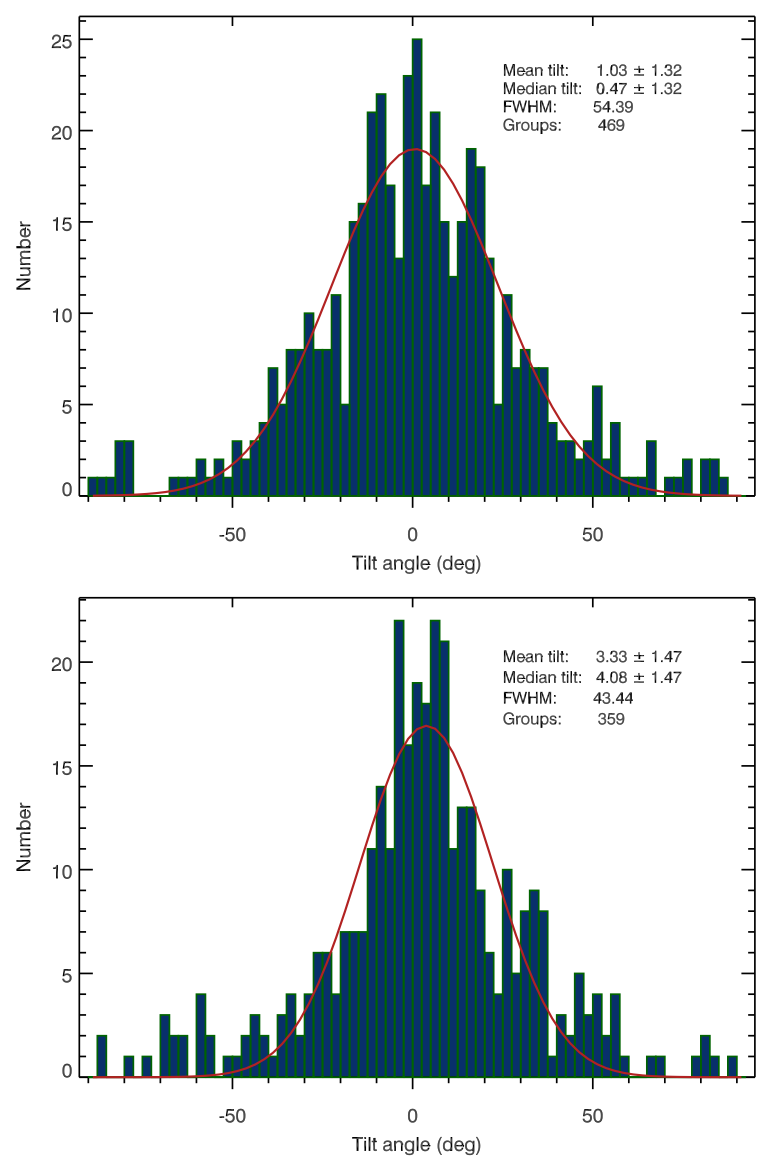

Figure 7: Tilt angle histograms for cycles 0 and 1 (top) and cycles 2, 3 and 4 (bottom) from Staudacher. Only groups with area weighted centers within $\pm 60^{\circ} \mathrm{CMD}$, polarity separations $\Delta \beta>3^{\circ}$ and with quality flags 1 and 2 are used.

all the tilt angles in the present paper is given by Senthamizh Pavai et al. (2015), where it was applied to the Schwabe data.

Friedrich Wilhelm Gustav Spörer observed sunspots from Anklam and Potsdam, Germany, during 1861-1894. He drew the sunspot groups while they crossed the central meridian, so the evolution of sunspot groups is not available. The details of the drawings and the technique used in the extraction of positions and areas of sunspots were given by Diercke et al. (2015).

\section{Tilt angle distributions}

Based on the positions of the individual spots, a tangential plane is adopted touching the solar surface in the group center in order to minimize curvature effects in determining the tilt angle. The groups are divided into two polarities such that the variance of the spot positions in the individual polarities are lowest. The method is explained in detail in Senthamizh Pavai et al. (2015). The sign of the tilt angle is positive when the leading polarity is closer to the equator than the following polarity. There is no sign of the polarities available from the sunspot drawings. Therefore, groups not obeying Hale's polarity law cannot be detected.

The definition of what forms a sunspot group varies among the various observers. We have therefore inspected all the data sets used here to re-group sunspots where it appeared necessary. We used the spot distributions as well as the evolution of the groups to discriminate them. The following numbers refer to groups through their full life-time as a single group, not to individual appearances of groups in each drawing. While the re-grouping for Schwabe is described in Senthamizh Pavai et al. (2015), a number of groups were split or combined in the records of the other observers as well. This procedure led to 13 new groups in Scheiner's drawings, whereas in seven cases, groups were combined to one. In the drawings by Hevelius, we obtained five new groups and combined groups in three cases. Zucconi's drawings contained two groups which needed to be split into two new groups each. Finally, 104 new groups were formed in Spörer's data, while in 90 cases, two groups were combined to a single group. Since Staudacher did not provide any group designations, the association of spots to groups was made from scratch by inspection of the drawings.

From those sunspot groups, the bipolar ones need to be extracted for the computation of tilt angles. The sunspot drawings of Scheiner, Hevelius, and Zucconi were manually inspected to select the bipolar groups, and only those groups are included in the present study. Figures 1, 2, and 4 show the distributions of tilt angles from the drawings of Scheiner, Hevelius, and Zucconi, respectively. In the distributions, only the bipolar groups within $\pm 60^{\circ}$ central meridian distance (CMD) are included, because the positional accuracy drops significantly beyond these limits. In all the graphs, we fit the discrete distributions with Gaussian functions to obtain estimates for the full widths at half-maximum (FWHM).

In case of Staudacher, Schwabe, and Spörer (Figs. 3, 5 , and 6 , respectively), the volumes of sunspot drawings were too large to manually pick the bipolar groups. For Staudacher and Schwabe, we complemented the condition of limiting the groups within $\pm 60^{\circ} \mathrm{CMD}$ by a minimum polarity separation $(\Delta \beta)$ of $3^{\circ}$ (Baranyi, 2015) in order to statistically remove unipolar groups. Figure 6 shows the tilt angle distribution of sunspot groups from Spörer's observations. Since the groups in Spörer's drawings are already at central meridian, only the condition $\Delta \beta>3^{\circ}$ was applied to remove potentially unipolar groups. The averages and medians of all the above tilt angle distributions are given in Table 1.

The FWHM for the tilt angle distributions are in the range of $30^{\circ}-40^{\circ}$, with one exceptional value of $50^{\circ}$ for Staudacher. Wang et al. (2015) obtained an FWHM of $30.8^{\circ}$ for the distribution of tilt angles from the Debrecen data using umbral data alone, as we do here as well.

The sizes of the sunspots in Staudacher's drawings are 
Table 2: Average and median tilt angles for individual solar cycles or small groups of cycles as well as the FWHM of the individual distributions. We use the cycle number notation of Zolotova \& Ponyavin (2015). Parentheses indicate that the cycle was not fully covered by observations.

\begin{tabular}{|c|c|c|c|c|c|}
\hline Data source & Cycle & Mean tilt & Median tilt & FWHM & Groups \\
\hline Scheiner & -12 (first half) & $\left(3.92^{\circ} \pm 0.91^{\circ}\right)$ & $\left(3.63^{\circ} \pm 0.91^{\circ}\right)$ & $\left(33.4^{\circ}\right)$ & 537 \\
\hline Hevelius & -10 (maximum) & $\left(4.79^{\circ} \pm 1.43^{\circ}\right)$ & $\left(5.35^{\circ} \pm 1.43^{\circ}\right)$ & $\left(27.1^{\circ}\right)$ & 130 \\
\hline Zucconi & $0+1(\min$. between 0 and 1$)$ & $\left(0.62^{\circ} \pm 2.28^{\circ}\right)$ & $\left(-1.48^{\circ} \pm 2.28^{\circ}\right)$ & $\left(35.8^{\circ}\right)$ & 131 \\
\hline \multirow[t]{2}{*}{ Staudacher } & $0+1$ & $1.03^{\circ} \pm 1.32^{\circ}$ & $0.47^{\circ} \pm 1.32^{\circ}$ & $54.4^{\circ}$ & 469 \\
\hline & $2+3+4$ & $3.33^{\circ} \pm 1.47^{\circ}$ & $4.08^{\circ} \pm 1.47^{\circ}$ & $43.4^{\circ}$ & 359 \\
\hline \multirow[t]{4}{*}{ Schwabe } & 7 (second half) & $\left(3.24^{\circ} \pm 0.55^{\circ}\right)$ & $\left(3.12^{\circ} \pm 0.55^{\circ}\right)$ & $\left(41.0^{\circ}\right)$ & 2243 \\
\hline & 8 & $4.36^{\circ} \pm 0.47^{\circ}$ & $5.05^{\circ} \pm 0.47^{\circ}$ & $42.9^{\circ}$ & 3419 \\
\hline & 9 & $4.71^{\circ} \pm 0.34^{\circ}$ & $4.79^{\circ} \pm 0.34^{\circ}$ & $40.3^{\circ}$ & 4942 \\
\hline & 10 & $4.74^{\circ} \pm 0.36^{\circ}$ & $4.94^{\circ} \pm 0.36^{\circ}$ & $39.7^{\circ}$ & 4898 \\
\hline \multirow[t]{4}{*}{ Spörer } & 10 (end) & $\left(2.91^{\circ} \pm 1.15^{\circ}\right)$ & $\left(1.77^{\circ} \pm 1.15^{\circ}\right)$ & $\left(19.5^{\circ}\right)$ & 465 \\
\hline & 11 & $4.59^{\circ} \pm 0.76^{\circ}$ & $4.59^{\circ} \pm 0.76^{\circ}$ & $32.0^{\circ}$ & 1067 \\
\hline & 12 & $5.73^{\circ} \pm 0.80^{\circ}$ & $4.63^{\circ} \pm 0.80^{\circ}$ & $34.3^{\circ}$ & 833 \\
\hline & 13 (beginning) & $\left(5.85^{\circ} \pm 1.11^{\circ}\right)$ & $\left(5.64^{\circ} \pm 1.11^{\circ}\right)$ & $\left(32.6^{\circ}\right)$ & 469 \\
\hline
\end{tabular}

highly exaggerated, so the area values were not used in the tilt angle calculation. Spörer magnified the sizes of sunspots in his drawings to some extent, and we needed to scale down the area values by a factor of 13.3 as inferred by Diercke et al. (2015). Spörer recorded both pores and umbrae but other drawings only contain umbrae or umbrae and penumbrae. To make it consistent with other data, only umbrae with areas $\geq 1 \mathrm{MSH}$ were considered while calculating the tilt angle.

\subsection{Comparison of cycle-averaged tilt angles}

The cycle-averaged tilt angle is a quantity related to the polar field generated by active regions in the course of a cycle and may be an indication of the activity of the future cycle. These averages are available since solar cycle 15 from modern data (e.g. Dasi-Espuig et al., 2010; McClintock \& Norton, 2013; Wang et al., 2015). Since historical data provide tilt angles over a much longer period, we look at cycle-averaged tilt angle values from Staudacher, Schwabe, and Spörer data which cover the cycles $0-4$ and 7-13. The data by Scheiner and Hevelius are not covering entire solar cycles. Their average may only be a rough indication of the cycle-average tilt angle and need to be treated with caution.

The Staudacher data covers solar cycles 0-4 without the beginning of cycle 0 . Table 1 shows that the mean tilt in the Staudacher and Zucconi data are lower than the ones from other data sources. While Staudacher's drawings are not very precise and could contain a strong random component bringing the average tilt angle close to zero, Zucconi's data are precise enough and confirm the very low average tilt. The data by Zucconi cover the cycle minimum between solar cycles 0 and 1 (February 1755 according to Hathaway, 2010) showing spots at latitudes from $0^{\circ}$ to $30^{\circ}$, apparently including spots from both the ceasing cycle 0 and the growing cycle 1 .

The butterfly diagram from the Staudacher data for the solar cycles 0 and 1 also shows a peculiar behaviour with an excess of groups at the equator (Arlt, 2009). We therefore divide the Staudacher data into two parts, such that one part contains the cycles 0 and 1 and the other part contains the remaining data (cycles 2-4), instead of dividing the data into individual cycles. The data do not contain the beginning of the cycle 0 but together with cycle 1 , we consider the result sufficiently representative for a two-cycle average. The mean tilt angles were found to be $1.03^{\circ} \pm 1.32^{\circ}$ for cycles 0 and 1 and $3.33^{\circ} \pm 1.47^{\circ}$ for cycles $2-4$. Figure 7 shows the tilt angle distributions separately for cycles 0 and 1 and the remaining part of the data. Using only the data with quality flag of 1 (disk orientation obtained from rotational matching of spots on adjacent days), the distinction between the two types of cycles is even clearer: the mean tilt for cycles 0 and 1 is $0.24^{\circ} \pm 1.61^{\circ}$, and it is $5.68^{\circ} \pm 1.83^{\circ}$ for the cycles $2-4$. The mean tilt angle for the cycles 0 and 1 is lower and on par with the mean tilt of Zucconi's data. The combined mean tilt for cycles 2-4 from the Staudacher data are higher and compatible with values of modern data. While the Staudacher data alone may be too inaccurate, the agreement with Zucconi's data is an indication for the peculiarity of the cycles 0 and 1.

The Schwabe data comprise cycles $7-10$, while the Spörer data cover cycles 10-13. The data for the initial two years of cycle 7 are missing in the Schwabe data, however. Data from Spörer are also not available for the first half of cycle 10 and the second half of cycle 13 . The spots being located at predominantly very low latitudes at the end of cycle 10 cause an underestimate of the mean tilt angle, according to Joy's law. Table 2 lists the mean and 
median tilt values for a number of cycles in the period 1620-1890. The FWHM were again derived from fits with Gaussian distributions. The average tilt for cycles 0 and 1 are the lowest of all solar cycles for which we have analysed drawings so far.

\section{Conclusions}

The various historical sunspot observations by Scheiner, Hevelius, Staudacher, Zucconi, Schwabe, and Spörer offer white-light sunspot drawings during the $17^{\text {th }}$, $18^{\text {th }}$, and $19^{\text {th }}$ centuries. The tilt angles for the supposedly bipolar sunspot groups from those different sunspot observations were calculated. The values from the period before the Maunder minimum (years in the 1620s and 1640s) are comparable to precise $20^{\text {th }}$-century results. The importance of the tilt angles for the transport of magnetic flux on the surface and the polar field was first noticed by Leighton (1964). Various effects of averaged tilt angles have been studied more recently (cf. e.g. Baumann et al., 2004; Cameron et al., 2010). The fact that the preMaunder minimum average tilt angles are relatively large suggests that it was not particularly low values that initiated a period of very low activity. If flux-transport dynamos are indeed operating in the Sun, more subtle effects such as, e.g., the occurrence of equator-crossing groups (Cameron et al., 2013) or other group properties need to be studied to find precursors for the very deep minimum following cycle -10 .

The cycle-averaged tilt angle values were also calculated for the solar cycles 0-4 and 7-13 from Staudacher, Schwabe and Spörer data. The mean tilt value for the cycles 0 and 1 seems to be the lowest of all cycle-averaged tilt angles, independently shown from sunspot drawings by Staudacher and precise images made by Zucconi. The accuracy of the Staudacher images may be questioned on the one hand, especially their orientation, but the agreement with Zucconi's data, on the other hand, is striking. The Sun also appeared to have a slightly stronger differential rotation in that period as compared to today (Arlt \& Fröhlich, 2012), but the result was not significant. Since this period is about three to four cycles after the Maunder minimum, it is not clear whether the features still represent the recovery from a grand activity minimum. While that early Staudacher period needs careful future inspection, the cycles before and after the Maunder minimum are clearly very valuable in constraining which kind of dynamo is operating in the Sun.

\section{Acknowledgements}

VSP acknowledges support by grant AR 355/10-1, CD acknowledges support by grant DE $787 / 3-1$, both of the German Science Foundation (DFG). JMV acknowledges support by grants GR15137 and AYA2014-57556-P.

\section{References}

Arlt, R., Digitization of sunspot drawings by Staudacher in 1749 1796, SoPh, 247, 399-410, 2008.

Arlt, R., The Butterfly Diagram in the Eighteenth Century, SoPh, 255, 143-153, 2009.

Arlt, R., The sunspot observations by Samuel Heinrich Schwabe, Astron. Nachr., 332, 805-814, 2011.

Arlt, R., Fröhlich, H.-E., The solar differential rotation in the 18th century, A\&A, 543, A7, 6pp., 2012.

Arlt, R., Leussu, R., Giese, N., Mursula, K., Usoskin, I.G., Sunspot positions and sizes for 1825-1867 from the observations by Samuel Heinrich Schwabe, MNRAS, 433, 3165-3172, 2013.

Baranyi, T., Comparison of Debrecen and Mount Wilson/Kodaikanal sunspot group tilt angles and the Joy's law, MNRAS, 447, 1857$1865,2015$.

Baumann, I., Schmitt, D., Schüssler, M., Solanki, S.K., Evolution of the large-scale magnetic field on the solar surface: A parameter study, A\&A, 426, 1075-1091, 2004.

Caligari, P., Moreno-Insertis, F., Schüssler, M., Emerging flux tubes in the solar convection zone. I. Asymmetry, tilt, and emergence latitude, ApJ, 441, 886-902, 1995.

Cameron, R.H., Jiang, J., Schmitt, D., Schüssler, M., Surface flux transport modeling for solar cycles 15-21: effects of cycledependent tilt angles of sunspot groups, ApJ, 719, 264-270, 2010.

Cameron, R.H., Dasi-Espuig, M., Jiang, J., Işık, E., Schmitt, D., Schüssler, M., Limits to solar cycle predictability: Crossequatorial flux plumes, A\&A, 557, A141, 6pp., 2013.

Charbonneau, P., Dynamo models of the solar cycle, Living Rev. Solar Phys., 7, 3-91, 2010.

Clette, F., Svalgaard, L., Vaquero, J.M., Cliver, E.W., Revisiting the sunspot number. A 400-year perspective on the solar cycle, Space Sci. Rev. 186, 35-103, 2014.

Cristo, A., Vaquero, J.M., Sánchez-Bajo, F., HSUNSPOTS: a tool for the analysis of historical sunspot drawings, JASTP, 73, 187190, 2011.

Dasi-Espuig, M., Solanki, S.K., Krivova, N.A., Cameron, R., Peñuela, T., Sunspot group tilt angles and the strength of the solar cycle, A\&A, 518, A7, 10pp., 2010.

Dasi-Espuig, M., Solanki, S.K., Krivova, N.A., Cameron, R., Peñuela, T., Sunspot group tilt angles and the strength of the solar cycle (corrigendum), A\&A, 556, C3, 2013.

Diercke, A., Arlt, R., Denker, C., Digitization of sunspot drawings by Spörer made in 1861-1894. Astr. Nachr., 336, 53-62, 2015.

D'Silva, S., Choudhuri, A.R., A theoretical model for tilts of bipolar magnetic regions, A\&A, 272, 621-633, 1993.

Fan, Y., Fisher, G.H., Radiative heating and the buoyant rise of magnetic flux tubes in the solar interior, SoPh, 166, 17-41, 1996.

Hathaway, D.H., The solar cycle, Living Rev. Solar Phys., 7, 1-65, 2010.

Hevelius, J., Selenographia sive, lunæ descriptio, Hünefeld, Gdańsk, 563 pp., 1647

Kosovichev, A.G., Stenflo, J.O., Tilt of emerging bipolar magnetic regions on the Sun. ApJL, 688, L115-L118, 2008.

Leighton, R.B., Transport of magnetic fields on the Sun, ApJ, 140, 1547-1562, 1964.

Li, J., Ulrich, R.K., Long-term measurements of sunspot magnetic tilt angles, ApJ, 758, 115, 12pp., 2012.

McClintock, B.H., Norton A.A., Recovering Joy's law as a function of solar cycle, hemisphere, and longitude, SoPh, 287, 215-227, 2013.

Scheiner, C., Rosa ursina sive sol, Andrea Fei, Bracciano, 822 pp., 1630.

Senthamizh Pavai, V., Arlt, R., Dasi-Espuig, M., Krivova, N.A., Solanki, S.K., Sunspot areas and tilt angles for solar cycles 7-10, A\&A, 584, A73, 13 pp., 2015.

Spörer, G., Ueber die Periodicität der Sonnenflecken seit dem Jahre 1618. In: Nova Acta der Ksl. Leop.-Carol. Deutschen Akademie der Naturforscher, Vol. 8, No. 2, 283-324, 1889.

Tian, L., Liu, Y., Wang, H., Latitude and magnetic flux dependence of the tilt angle of bipolar regions, SoPh, 215, 281-293, 2003. 
Usoskin, I.G., Arlt, R., Asvestari, E., Hawkins, E., Käpylä, M. et al., The Maunder minimum (1645-1715) was indeed a grand minimum: A reassessment of multiple datasets, A\&A, 581, A95, 19 pp., 2015.

Wang, Y.-M., Colaninno, R.C., Baranyi, T., Li, J., Active-region tilt angles: magnetic versus white-light determinations of Joy's law, ApJ, 798, 50, 14 pp., 2015.

Weber, M.A., Fan, Y., Miesch, M.S., The rise of active region flux tubes in the turbulent solar convection zone, ApJ, 741, 11, 14 pp., 2011.

Weber, M.A., Fan, Y., Miesch, M.S., Comparing simulations of rising flux tubes through the solar convection zone with observations of solar active regions: constraining the dynamo field strength, SoPh, 287, 239-263, 2013.

Zolotova, N.V., Ponyavin, D.I., The Maunder minimum is not as grand as it seemed to be, ApJ, 800, 42, 14 pp., 2015. 